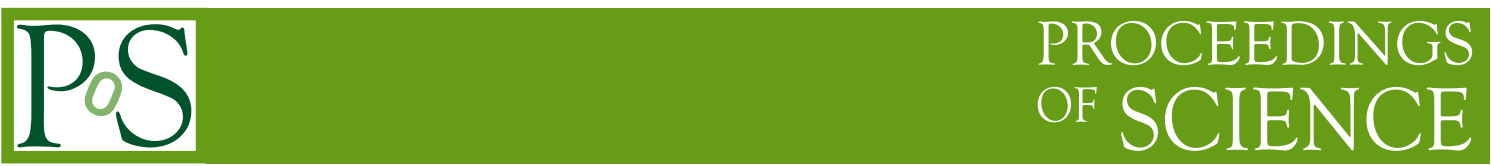

\title{
Charge-starved jets and rapid variability in blazars
}

\author{
Iwona Mochol and John G. Kirk \\ Max-Planck-Institut für Kernphysik, Postfach 1039 80, 69029 Heidelberg, Germany \\ E-mail: iwona.mochol@mpi-hd.mpg.de, john.kirk@mpi-hd.mpg.de
}

\begin{abstract}
Very rapid variations of the gamma-ray flux from blazars suggest that there is a mechanism at work which modulates blazar emission on timescales much smaller than the light-crossing time of the black hole's event horizon. We propose a scenario in which blazar photons are modulated at the frequency $\omega$ of a large-amplitude wave that is launched in the polar region of the central, rotating black hole, and propagates in a charge-starved jet. Using a two-fluid $\left(e^{ \pm}\right)$description, we find the outflow exhibits a delayed acceleration phase that starts when the inertia associated with the wave currents becomes important. The modulation of the emission from the accelerating jet is preserved for the observer provided that the density of pairs, produced in an electromagnetic cascade, is sufficiently low.
\end{abstract}

25th Texas Symposium on Relativistic Astrophysics - TEXAS 2010

December 06-10, 2010

Heidelberg, Germany 


\section{Introduction}

H.E.S.S. observations of PKS 2155-304 show remarkably rapid flux variations on a timescale $\sim 2-5$ minutes $[1,6]$, which corresponds to a spatial structure that is roughly one hundred times smaller than the gravitational radius $r_{\mathrm{g}}=G M / c^{2}$ of the central black hole. These small structures modulate the entire energy flux reaching the observer, which suggests that they are located close to, or even within, the black-hole magnetosphere. In our model [9] we associate them with the wavelength $2 \pi c / \omega$ of a nonlinear electromagnetic wave that propagates radially in the jet direction, and, in fact, constitutes the jet itself. In an astrophysical context, nonlinear electromagnetic waves were originally discussed in connection with pulsars [3], but the emphasis was placed on modes with superluminal phase speeds that can propagate only in a very low density plasma. The mode we study here, on the other hand, has a subluminal phase speed, and propagates also in the relatively dense environment that can be expected close to a pulsar or black hole [8]. Our treatment is limited to radially propagating plane waves, i.e., we study the waves when they are already far from the place where they were generated, and assume their lateral extent is large compared to their wavelength. Close to the wave's point of origin, its lateral extent is presumably comparable to its wavelength, which not only rules out a plane wave description, but also the invalidates the assumption of an axially symmetric jet. Thus, our model supposes that the waves are generated by a generalised, non-axisymmetric form of the familiar axisymmetric Blandford-Znajek mechanism [4]; one which causes the inhomogeneous magnetosphere of a rotating black hole to drive a Poynting-flux-dominated jet. Some part of this jet will propagate in the low-density funnel that is both expected on theoretical grounds [18] and observed in numerical simulations [5, 15]. Accreting plasma cannot penetrate into this funnel. The matter density there is likely to be dominated by electron-positron pairs that materialise within the magnetosphere, in a manner analogous to that which operates in a pulsar magnetosphere.

\section{Jet parameters}

The physical conditions in a pair-dominated funnel-jet are determined by three dimensionless parameters:

1. The energy flux density is measured by the strength parameter $a=a_{0}(c / \omega r)$, where

$$
a_{0}=\left[4 \pi e^{2} L /\left(m^{2} c^{5} \Omega_{\mathrm{s}}\right)\right]^{1 / 2}
$$

with $L$ the luminosity carried in a solid angle $\Omega_{\mathrm{s}}$. Physically, $a$ is the conventionally defined strength parameter of a circularly polarised vacuum electromagnetic wave that carries the same energy flux density as the jet.

2. The mass-loading of the jet is defined by $\mu=L / \dot{M} c^{2}$ [16], with $\dot{M}$ the mass flux carried by the jet, which is determined not by accretion, but by the production of pairs in an electromagnetic cascade. Alternatively, one can use the parameter $\kappa$, the pair multiplicity that is conventionally used in pulsar physics, and specify it through its value $\kappa_{r_{\mathrm{g}}}$ at the fiducial radius $r_{\mathrm{g}}$ :

$$
\kappa_{r_{\mathrm{g}}} \approx \frac{a_{0}}{4 \mu}\left(\frac{c}{\omega r_{\mathrm{g}}}\right)
$$


(e.g., [14]).

3. The magnetisation parameter $\sigma$ which describes the ratio of the energy flux carried by electromagnetic fields to that carried by particles. For a cold, radially flowing jet with Lorentz factor $\gamma$,

$$
\sigma=(\mu / \gamma)-1
$$

Such a flow is super-magnetosonic provided $\sigma<\sigma_{0}=\mu^{2 / 3}$.

\section{The Model}

As is well-known (e.g., [17]), a cold, collisionless plasma can be described by the equations of ideal MHD, provided waves of frequency comparable to or higher than the plasma frequency can be neglected. In a relativistic, supersonic jet, this is widely thought to be the case. (In fact, although a rigorous theoretical justification is absent, ideal MHD is also routinely applied to collisionless flows that are warm and subsonic.) Such a plasma supports several low-frequency wave modes, in which, because of the assumption of perfect conductivity, the current is given simply by the curl of the magnetic field, independent of the plasma speed and density.

For small amplitude waves in a sufficiently dense plasma this approximation works well. However, since the outflow of a pair plasma in a funnel-jet may carry a large amplitude wave and relatively few particles, i.e., can be charge-starved in our terminology, the ideal MHD description of the jet may not be adequate [11]. Therefore, we adopt an approach that allows us to take into account non-MHD effects connected with the finite inertia of the charge-carriers: the jet is described as two cold, relativistic $e^{ \pm}$fluids. For the low frequency waves we consider, this model can be simplified even further. It suffices to introduce the number density and radial momentum which are the same for each fluid: $n_{+}=n_{-}=n, p_{\|_{+}}=p_{\|_{-}}=p_{\hat{r}}$, and the (complex) transverse or perpendicular momenta, which are of opposite sign: $p_{\perp+}=-p_{\perp_{-}}=p_{\hat{\theta}}+i p_{\hat{\phi}}$. (These dimensionless quantities are given in units of $m c$.) This notation is particularly convenient when the fluids support a transverse electromagnetic wave that is circularly polarized, with (complex) electric and magnetic fields $E=E_{\hat{\theta}}+i E_{\hat{\phi}}$ and $B=B_{\hat{\theta}}+i B_{\hat{\phi}}$. In the following we study the fate of a large-amplitude wave of this kind that is launched from the polar regions of a black hole magnetosphere, and propagates radially.

\section{Subluminal waves}

The equations to be solved are the continuity equations and the equations of motion for each fluid, together with Faraday's and Ampere's laws. (For transverse fields and currents, and zero charge-density, the other two Maxwell equations are automatically satisfied.) To study nonlinear waves, we follow the approach introduced by [2]. For plane waves, this entails expressing all quantities in terms of a single phase variable $\phi$. In order to follow the evolution of these waves over large distances (compared to a wavelength) in spherical geometry, we introduce a radial coordinate $r$, that varies slowly compared to $\phi$, and expand in the small parameter $\varepsilon \sim c / \omega r \ll 1$ (short wavelength approximation). In addition, all the equations are formulated in a Kerr metric, following 
[7], and expanded in $\varepsilon \sim r_{g} / r \ll 1$, i.e., assuming the gravitational radius is of the same order in the small parameter as the wavelength. In the application to PKS 2155-304, the wavelength may be one hundred times smaller than $r_{g} \sim c / \omega$, but this factor is not large compared to the value of $1 / \varepsilon$ at the distances we consider (typically $\sim 10^{4} r_{\mathrm{g}}$ ). It turns out that to first-order in $\varepsilon-$ which is sufficient to determine the wave evolution — general relativistic effects drop out of the equations.

The WKB-like approach in which the solution is assumed to vary much faster in the phase variable than in the radial variable, allows us to obtain the properties of a wave from the zerothorder equations, which also describe plane waves. These equations are: the continuity equation

$$
\omega \frac{\partial}{\partial \phi}(n \Delta)=0
$$

Faraday's and Ampère's laws:

$$
\begin{aligned}
-\frac{\omega}{\beta_{\mathrm{w}}} \frac{\partial E}{\partial \phi}-i \omega \frac{\partial B}{\partial \phi} & =0 \\
-\frac{\omega}{\beta_{\mathrm{w}}} \frac{\partial B}{\partial \phi}+i \omega \frac{\partial E}{\partial \phi}+i 8 \pi e n p_{\perp} & =0
\end{aligned}
$$

and the momentum/energy equations:

$$
\begin{aligned}
\omega \Delta \frac{\partial p_{\|}}{\partial \phi}+\frac{e}{m} \operatorname{Im}\left(p_{\perp} B^{*}\right) & =0 \\
\omega \Delta \frac{\partial p_{\perp}}{\partial \phi}-\frac{e}{m}\left(\gamma E+i p_{\|} B\right) & =0 \\
\omega \Delta \frac{\partial \gamma}{\partial \phi}-\frac{e}{m} \operatorname{Re}\left(p_{\perp} E^{*}\right) & =0,
\end{aligned}
$$

where $\Delta=\gamma-p_{\|} / \beta_{\mathrm{w}}, \gamma=\sqrt{1+p_{\|}^{2}+\left|p_{\perp}\right|^{2}}$, and $\beta_{\mathrm{w}}$ is the phase velocity of the wave.

These equations possess a subluminal solution $\left(\beta_{\mathrm{w}}<1\right)$ in which the wave is in resonance with the fluids $(\Delta=0)$. In the frame comoving with the wave, the electric field vanishes and the wave is a static magnetic field of constant magnitude whose direction rotates through $2 \pi$ radians over one wavelength. At each point, $p_{\perp}$, and, hence, the plasma current, is parallel to the magnetic field, so that no force is exerted on the fluids. In fact, the rate at which the $B$-vector rotates is arbitrary, being determined by the dependence of the fluid density $n$ on phase. We consider the simplest case, where $n,|B|^{2}$ and $\left|p_{\perp}\right|^{2}$ are all constant and the wave is a monochromatic magnetic shear: $B \propto p_{\perp} \propto \mathrm{e}^{ \pm i \phi}$.

The slow, radial evolution of this plane-wave solution is described by the first-order equations. In the standard manner we eliminate secular terms in the first-order quantities by demanding their periodicity in $\phi$, and obtain three independent equations: the first order continuity equation

$$
\frac{1}{r^{2}} \frac{\partial}{\partial r}\left(r^{2} n p_{\|}\right)=0
$$

the conservation of the phase-averaged energy flux

$$
\frac{\partial}{\partial r}\left[r^{2}\left(p_{\|} n \gamma+\frac{\beta_{\mathrm{w}}|B|^{2}}{8 \pi m}\right)\right]=0
$$


and the momentum equation

$$
\frac{\partial}{\partial r}\left[r^{2}\left(p_{\|}^{2} n+\left(1+\beta_{\mathrm{w}}^{2}\right) \frac{|B|^{2}}{16 \pi m}\right)\right]=r\left|p_{\perp}\right|^{2} n .
$$

(Note that the term on the RHS of (4.9) was erroneously omitted in [9].) The solution, which is easily found numerically, reveals three phases: These are, as functions of the dimensionless radius $R=\mu r \omega /\left(a_{0} c\right)$ :

1. The coasting phase, where $R \ll \mu / \sigma_{0}$. Here the inertia of current carriers is negligible and the supersonic $\left(\sigma_{0}<\mu^{2 / 3}\right)$, relativistic flow coasts at constant speed and magnetisation, and can be described using single-fluid, ideal MHD

$$
p_{\perp} \ll 1 \quad \gamma \approx \gamma_{\mathrm{w}} \approx \mu / \sigma_{0}
$$

2. The acceleration phase, where $\mu / \sigma_{0} \ll R \ll \mu$. Here, the inertia associated with current contributes significantly to the energy-momentum flux

$$
p_{\perp} \approx 1 \quad \gamma \approx \gamma_{\mathrm{w}} \approx R, \quad \sigma \approx \mu / R
$$

3. The free-streaming phase, where $R \gg \mu$. Here the electromagnetic fields are negligible and the cold plasma streams outwards at constant speed.

$$
\gamma \approx \gamma_{\mathrm{w}} \approx \mu, \quad\left|p_{\perp}\right| \ll 1
$$

This solution is illustrated in Fig. 1.

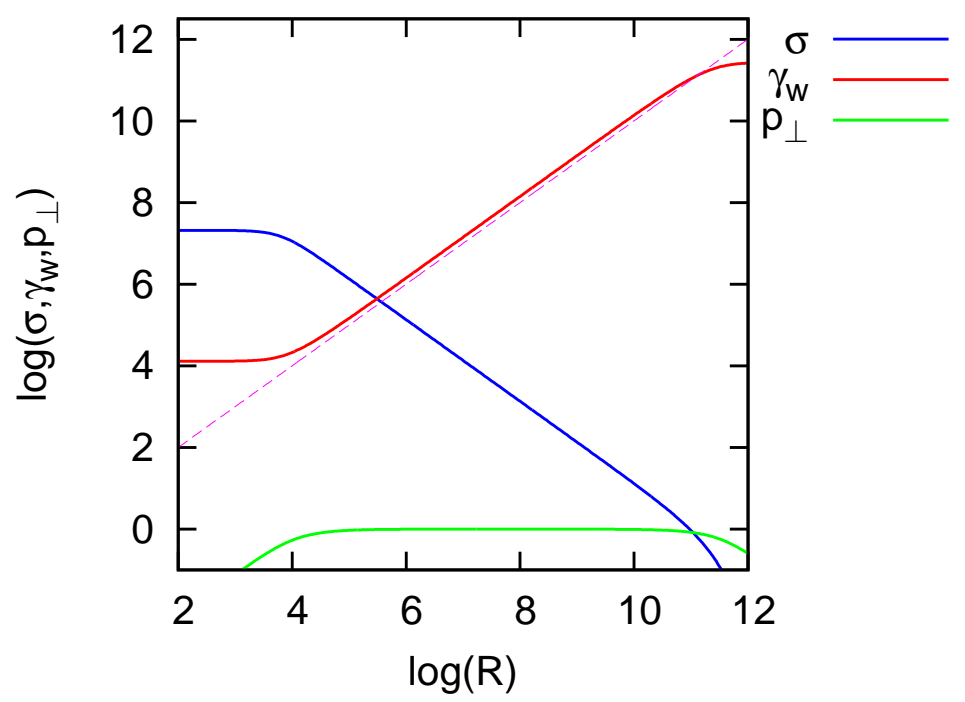

Figure 1: The solution as a function of the dimensionless radius $R=\mu r \omega /\left(a_{0} c\right)$, plotted for $\mu=2.7 \times 10^{11}$ and $\sigma_{0}=0.5 \mu^{2 / 3}=2.1 \times 10^{7}$, together with the approximation $\gamma_{\mathrm{w}}=R$ 


\section{Application to blazar variability}

The blazar PKS 2155-304 exhibits substantial variations on a time scale of roughly $100 \mathrm{~s}$ and at a flux level that corresponds to an "isotropic" or " $4 \pi$ " luminosity $\left(=4 \pi L / \Omega_{\mathrm{S}}\right)$ of roughly $10^{46} \mathrm{erg} \mathrm{s}^{-1}$. If the variation timescale in units of $100 \mathrm{~s}$ is denoted by $\Delta t_{100}=(2 \pi / \omega) /(100 \mathrm{~s})$, and the $4 \pi$-luminosity in units of $10^{46} \mathrm{erg} \mathrm{s}^{-1}$ by $L_{46}$, the jet parameters are

$$
a_{0}=3.4 \times 10^{14} L_{46}^{1 / 2}, \quad \omega=0.06 \mathrm{~s}^{-1} \Delta t_{100}^{-1}
$$

and, thus,

$$
\mu=2.7 \times 10^{11} \Delta t_{100} \kappa_{r_{g}}^{-1} L_{46}^{1 / 2} M_{9}^{-1} .
$$

According to (4.12), the value of $\mu$ equals the final Lorentz factor of the particles in the freestreaming phase. Since, in blazars, $\mu$ is very large, we can expect that processes we have neglected intervene well before this phase is reached. These processes might include inverse-Compton upscattering of ambient soft photons, interactions with the external medium, and instabilities intrinsic to the two-fluid system.

In the particular solution we consider here, the particles move radially in resonance with the wave and their radiation should therefore be modulated at the wave frequency $\omega$. The criterion that the modulations at this frequency of the jet emission are not washed out by the variation of the travel time of the signal across the source is

$$
\gamma_{\mathrm{w}}^{2} 2 \pi c / \omega>r
$$

([10]). For this to be satisfied throughout the acceleration phase, it suffices that it is satisfied at the point $r=r_{\text {acc }}=a_{0} c /\left(\omega \sigma_{0}\right)$, where this phase starts. This is because $\gamma_{\mathrm{w}}$ is constant for $r<r_{\text {acc }}$ and $\gamma_{\mathrm{w}} \propto r$ for $r>r_{\mathrm{acc}}$, so that $\gamma_{\mathrm{w}}^{2} / r$ has its minimum value at $r=r_{\mathrm{acc}}$. Since

$$
r_{\mathrm{acc}} \approx 1.2 \Delta t_{100}^{1 / 3} \kappa_{r_{\mathrm{g}}}^{2 / 3} L_{46}^{1 / 6} M_{9}^{2 / 3} \mathrm{pc}
$$

and the Lorentz factor of the flow at this point is

$$
\gamma_{0} \approx 6.5 \times 10^{3} \Delta t_{100}^{1 / 3} \kappa_{r_{\mathrm{g}}}^{-1 / 3} L_{46}^{1 / 6} M_{9}^{-1 / 3}
$$

(5.3) gives an upper limit on the multiplicity:

$$
\kappa_{r_{\mathrm{g}}}<14 \Delta t_{100} L_{46}^{1 / 8} M_{9}^{-1}
$$

If our interpretation is correct, this inequality implies that extreme variability can be exhibited only by sources that do not develop prolific electromagnetic cascades. It is interesting to note that the magnetospheres of such sources should be able to support vacuum gaps [13]. In this case, in analogy with pulsar magnetospheres $[12,19]$, one can expect rapid variability to be generated by non-stationary gap discharges. Thus, rapid variability is not only possible for charge-starved sources, but it is also expected. 


\section{Summary and conclusions}

In this contribution, we study a particular, WKB-like solution of the non-linear equations that describe the propagation of large-amplitude, transverse, circularly polarized waves in a low-density pair plasma. Such a wave, when launched in the polar regions of a black-hole magnetosphere, propagates along the jet in resonance with particles. The radial evolution of the wave exhibits a delayed acceleration phase, which cannot be described within the ideal MHD approximation, since it occurs where the inertia of the current becomes important. Although we have demonstrated the existence of this phase for only one particular wave solution, the physics that underlies it suggests that it may be a generic phenomenon. When the particles in the jet start to radiate, their emission should be modulated at the wave frequency. We have shown that rapid modulations can be observed only from magnetospheres that can sustain vacuum gaps, and, therefore, harbour a natural source of the variations themselves.

\section{References}

[1] F. Aharonian et al. 2007, ApJ, 664, L71

[2] A.I. Akhiezer and R.V. Polovin 1956, Sov. Phys. JETP, 3, 696

[3] E. Asseo, F. C. Kennel, and R. Pellat 1975, A\&A, 44, 31

[4] R. D. Blandford \& R. L. Znajek 1977, MNRAS, 179, 433

[5] J. De Villiers \& J. F. Hawley 2003, ApJ, 592, 1060

[6] HESS Collaboration et al. 2010, A\&A, 520, A83

[7] R. Khanna 1998, MNRAS, 294, 673

[8] J. G. Kirk 2010, Plasma Physics \& Controlled Fusion, 52, 124029

[9] J. G. Kirk \& I. Mochol 2011, ApJ, 729, 104

[10] J. G. Kirk, O. Skjæraasen, Y. A. \& Gallant 2002, A\&A, 388, L29

[11] S. Koide 2009, ApJ, 696, 2220

[12] A. Levinson, D. Melrose, A. Judge \& Q. Luo 2005, ApJ, 631, 456

[13] A. Levinson \& F. Rieger 2011, ApJ, 730, 123

[14] Y. Lyubarsky \& J. G. Kirk 2001, ApJ, 547, 437

[15] J. C.McKinney \& C. F. Gammie 2004, ApJ, 611, 977

[16] F. C. Michel 1969, ApJ, 158, 727

[17] D. C. Montgomery \& D. A. Tidman 1964, Plasma Kinetic Theory, (New York: McGraw-Hill)

[18] K. S. Thorne, K. S., Price \& D. A. MacDonald 1986, Black Holes: The Membrane Paradigm, ed. K. S. Thorne, K. S., Price \& D. A. MacDonald (New Haven, CT: Yale Univ. Press)

[19] A. N. Timokhin 2010, MNRAS, 408, 2092 